\title{
BILLETS POUR VOYAGES CIRCULAIRES
}

\author{
(A PRIX RÉdutrs)
}

sur le réseau des chemins de fer d'Alsace-Lorralne.

Ces billets, établis pour faciliter la visite des Vosges, sont valables pendant 45 jours et délivrés toute l'année. Ils sont combinés pour être pris, au départ, dans les villes suivantes :

Strasbourg, Colmar, Mulhouse, Sarreguemines, Metz, Saverne, Morhange (Mörchingen), Thann, Rouffach, Bâle.

Ces billets peuvent comprendre dans leur itinéraire une interruption de parcours, correspondant à une traversée de montagne; ils mènent alors le voyageur jusqu'à la station terminus en amont d'une vallée, pour le reprendre à la station d'amont d'une vallée voisine.

Le détail des combinaisons, qui se montent à une soixantaine, se trouve dans l'édition allemande de ce Guide, et dans les indicateurs de chemins de fer.

\section{JALONNEMENT DES CHEMINS \\ DANS LES VOSGES \\ au moyen de marques de couleur.}

Suivant une décision du Club vosgien, on a fait choix d'une ronte ou arête principale marquée d'une couleur uniforme sur tout son parcours; on a marqué de même un certain nombre de routes voisines ou chemins ayant accès à la première.

La route principale, que nous appellerons dans ce volume : trajet principal, rectangle rouge, suit toute la chaine des Vosges, du Nord au Sud, commençant à Schœnau et finissant près de Sewen, après avoir touché les points principaux du pays, les plus dignes d'être visités.

Des routes secondaires, rectangle rouge avec bande blanche, conduisent à l'arête principale et mènent aux points intéressants qui se trouvent en dehors de celle-ci.

Des routes locales avec d'autres marques et d'autres couleurs (blanc, jaune, bleu-vert, etc.) sont désignées par les sections du Club, dans leur propre domaine et à leur gré.

Nous ne donnons pas ici la longue liste des chemins ainsi jalonnés et qui occupe 14 pages de la $\mathrm{X}^{\mathrm{e}}$ édition allemande. Les marques en couleur ètant fréquemment désignées dans le cours du livre, ces renseignements ont paru suffisants au traducteur. 